\title{
VEITSMAN'S THEOREM ON SPACES AND FORCES FORMING THEM, TIME AND CHAOS
}

\author{
Emil V. Veitsman *1凶 \\ ${ }^{* 1}$ Veitsman's Science Project, 28 department, 5 Klimashkin Street, 123557, Moscow, Russia
}

DOI: https://doi.org/10.29121/granthaalayah.v8.i6.2020.349

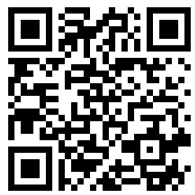

Article Type: Research Article

Article Citation: Emil V. Veitsman. (2020). VEITSMAN'S THEOREM ON SPACES AND FORCES FORMING THEM, TIME AND CHAOS. International Journal of Research GRANTHAALAYAH, 8(6), 26 - 29. https://doi.org/10.29121/granthaa layah.v8.i6.2020.349

Received Date: 01 May 2020

Accepted Date: 20 June 2020

Keywords:

Space

Forces

Universe

Time

Chaos

\section{ABSTRACT}

Some consequences follow from Veitsman's theorem. It is supposed that our physical time is a criterion characterizing the flow of any fundamental process. It is supposed as well that physical time in space defines from the state of the system under study, e.g., of Universe. If the space is in equilibrium then there is no time known to us from physics in it. The physical time appears in case when the energy expenses take place during the process flowing in space studied. If the space is anisotropic then the time is to be a tensor of rank 2 when its off-diagonal components equal zero. The anisotropy of the space and the change of the physical time passage lead to the breach of the causal connections in the system under study and, as a result, to chaos in it.

\section{INTRODUCTION}

Veitsman's theorem on spaces and forces says [1,2]: "Long-raged interaction forces of the dimensions $\mathbf{i}=0,1$, $2, \ldots, \mathbf{n}$ can form real isotropic Euclidean spaces if and only if the dimensions $\mathbf{j}$ of these spaces equal $\mathbf{j}=\mathbf{i}+1$ ".

This theorem was proved with help of the following expressions:

$$
\begin{aligned}
& \operatorname{div}_{n} \boldsymbol{A}_{(\mathbf{n})}=\operatorname{Lim} \frac{\oint_{n} a_{\rho} n_{\rho} d \Lambda_{(n-1)}}{\Omega_{(n)}} ; \Omega_{(n)}=C_{(n)} \rho^{n} . \Lambda_{(n-1)}=n C_{(n)} \rho^{n-1}, C_{(n)}=\frac{\pi^{n / 2}}{\Gamma\left(\frac{n}{2}+1\right)}, \\
& \Omega_{(n)} \rightarrow 0 \\
& \mathbf{F}_{(\mathrm{n})}=-k m_{1} m_{2} \mathbf{R}_{(\mathrm{n})} / R_{(n)}^{n} ; \mathbf{A}_{(\mathrm{n})}=\mathbf{E}_{(\mathrm{n})}=\left(\mathbf{F}_{(\mathrm{n})} / m_{2}\right)=-\mathbf{R}_{(\mathrm{n})} \frac{k m_{1}}{R_{(n)}^{n}} ;
\end{aligned}
$$


Where index " $n$ " in $(1-2)$ indicates that these formulae refer to $\mathbf{n D}$-spaces $(\mathbf{n}=1,2,3 \ldots \mathrm{m}) ; d \Lambda_{(n-1)}$ is the element of (n- 1)D-surface; $n_{\rho}$ the component of unit vector perpendicular to each point of this (n - 1)D-surface; $\Omega_{(n)}$ the $\mathbf{n D}$-volume; $\mathbf{F}_{(\mathrm{n})}$ the interaction force between of masses $m 1$ and 2 in $\mathbf{n D}$-space; $k$ the constant of gravitation in $\mathbf{n D -}$ space $\left(\mathrm{kg}^{-1} \cdot \mathrm{m}^{\mathrm{n}} \cdot \mathrm{s}^{-2}\right) ; \mathbf{E}$ the vector of gravitation field intensity $\left(\mathrm{m} \cdot \mathrm{s}^{-2}\right)$, as $m_{2}=1 ; \Gamma\left(\frac{n}{2}+1\right)$ the gamma function.

It was shown, if the theorem will not be respected then the space of each dimension $(\mathbf{n}=1,2,3, \ldots)$ cannot be formed. In turn, in [1] it was shown, if, e.g., space studied is weak anisotropic, then it will be existing only some time. A moment will occurs when the space ends to exist. However if there is no the physical space then there is no and physical time as well. It is evidently. A suspect occurs that the physical time is indissoluble connected with the space via the transformation of the latter. We will make an attempt to show it. It is the main goal of this short article,

\section{MATERIALS AND METHODS}

For electro-magnetic forces, namely, for Coulomb's law and the intensity (e) of them, the divergence of electromagnetic field for electric charges $q_{1(+)}$ and $q_{2(-)}$ can be written as:

$$
\begin{aligned}
& \operatorname{div}_{3} \mathbf{e}=\operatorname{Lim} \frac{\oint_{3} e_{\rho} n_{\rho} d S}{V}, V=\frac{4}{3} \pi r^{3}, S=4 \pi r^{2}, \\
& V \rightarrow 0, \\
& \mathbf{F}=-q_{1(+)} q_{2(-)} \mathbf{r} / \varepsilon r^{3} ; \mathbf{e}=\mathbf{E}=\left(\mathbf{F} / q_{2(-)}\right)=-\mathbf{r} \frac{q_{1(+)}}{\varepsilon r^{3}}
\end{aligned}
$$

Where index " 3 " in (3) indicates that the above formulae refer to 3D-space; $d S$ is the surface element of a spherical surface; $n_{\rho}$ the unit vector perpendicular to $d S$; $V$ the volume; $\mathbf{F}$ the interaction force between charges $q$ $\left(\mathrm{kg}^{1 / 2} \cdot \mathrm{m}^{-(3 / 2)} \cdot \mathrm{s}^{-1}\right) ; 1$ and $2 ; \varepsilon=1$, dielectric constant in vacuum; $\mathbf{E}$ the vector of electrical field intensity $\left(\mathrm{kg}^{1 / 2} \cdot \mathrm{m}^{5 / 2}\right.$ - $\mathrm{s}^{-1}$ ), as $q_{2}=1$.

Electro-magnetic interactions are more intensive than gravitational ones, however they are short-range. They can form a space only in separated volumes of our space-time therefore it is very doubtfully that unified field theory can be formulated. The short-range forces cannot influence on forming Universe. There is no physical time in Veitsman's theorem as a explicit quantity - the time is found there in latent form. As known, our space-time is expanding and it is isotropic regarding gravitational interaction and physical time. But what is the physical time?

We can touch to the linear sizes or, in any case, look at them, e.g., at the etalon of meter done from Iridium and

Platinum. Real substance is in the base of the linear sizes. But can we touch the physical time or look at it? No, of course. Such time is something imaginary. We can only state that the physical time is a criterion that characterizes a flow of physical processes. This criterion is closely bound with the energy spent in the process. We can define the physical time $t$ as

$$
t=\frac{\delta \mathrm{E}}{\delta \mathrm{M}}
$$

Where E is energy used in the process (J), M power of the process (W), and, that is, the physical time is not a postulate. Here it is a function from energy consumed in certain physical process.

However there are many physical processes in Universe but the physical time is only one according to our observations. This quantity $\mathrm{T}$ is a scalar. Then is there a fundamental process in our Universe which defines the physical time known from physics? We consider that it is the process of its (Universe) expanding. This process is stationary and central-symmetric in the space because our Universe is isotropic. Then we can write $\mathrm{T}$ as. 
$\mathrm{T}=C_{t} \frac{\delta \mathrm{E}}{\delta V}=$ const

Here $\mathrm{E}$ is the energy of the expansion of our Universe, $C_{t}$ a time constant $\left(\mathrm{s}^{3} \cdot \mathrm{m} \cdot \mathrm{kg}^{-1}\right)$. Namely the constancy of its (of the energy) specific density gives us the constancy of physical time, in other words, steady passage (step) of time. But what can we identify this energy with? We have to identify this energy with the energy of vacuum. Here the vacuum is a starting point.

Of course, there are many different processes in Universe but their total energy is much less that the vacuum energy. Therefore these processes cannot, as a rule, effect on the fundamental time of the Universe. If $\frac{\delta \mathrm{E}}{\delta V}=0$, then Universe is in equilibrium and there is no any main time. As a consequence, there is a time in each subspace depending on processes flowing in this part of the space.

In [1] we showed that the weakly anisotropic spaces cannot be in equilibrium therefore they have to change differently in different directions but then the physical time has also to change differently in different ones, i.e., physical time is tensor here, namely,

$$
\begin{aligned}
& \mathrm{T}_{i k}=\left(\begin{array}{ccc}
\mathrm{T}_{11} & 0 & 0 \\
0 & \mathrm{~T}_{22} & 0 \\
0 & 0 & \mathrm{~T}_{33}
\end{array}\right), \\
& \mathrm{T}_{11}=C_{(t) 1} \frac{\delta \mathrm{E}}{\delta V \varepsilon_{1}}, \\
& \mathrm{~T}_{22}=C_{(t) 2} \frac{\delta \mathrm{E}}{\delta V \varepsilon_{2}}, \\
& \mathrm{~T}_{33}=C_{(t) 3} \frac{\delta \mathrm{E}}{\delta V \varepsilon_{3}},
\end{aligned}
$$

Where $\varepsilon_{i}$ are the components of the space elongation in different direction in $\%$ from the volume $V$ of system under study.

From (7) - (10) it follows that causal relationships have to be broken in the anisotropic space, and a chaos must there begin. In turn, if instead (6) we shall have

$$
\mathrm{T}=C_{t} \frac{\delta \mathrm{E}}{\delta V} \neq \text { const }
$$

Then there will be another disorder in our space under study because the step of time will change and the flows of all processes, e.g., physical ones, will not be predictable.

\section{DISCUSSION}

As known, old people believe that time is running out for them faster than for young people. We consider that this phenomenon can be explained owing to the above results obtained. Indeed, there are many physical and chemical processes flow in "the system" named human being. Short range interactions lay in the base of them, e.g., electromagnetic ones. The electromagnetic interactions are much more intensive than gravitational one on the little distances and they can creature their very small subspaces in our Universe influencing on the physical main time in 
these small systems. Electromagnetic processes in this subspace can, in turn, generate a physical time which sum itself with the main physical time of our Universe. As a result, the physical time for the old people begins to overtake the calendar or, if you want, kinematic time.

\section{CONCLUSIONS}

The main conclusions are following.

1) We exist in the Universe which is isotropic and expands stationary.

2) We can exist only in such space-time.

3) If our space-time were anisotropic and would expand no stationary, then causal connections in Universe would break and chaos (a causal turbulence) would reign.

\section{SOURCES OF FUNDING}

None.

\section{CONFLICT OF INTEREST}

None.

\section{ACKNOWLEDGMENT}

None.

\section{REFERENCES}

[1] E.V. Veitsman. Mathematics \& Statistics 7(5), 197 (2019).

[2] E.V. Veitsman. Phys. Astron. Int. J. 2(4), 399 (2018). 